\title{
New Type of Guided Waves in Birefringent Media
}

\author{
Lluís Torner, Juan P. Torres, and Dumitru Mihalache
}

\begin{abstract}
Existence of waves guided by thin dielectric films deposited over a positive birefringent crystal for waveguide parameters below usual cutoff is discussed. This additional kind of guided waves have a hybrid nature and occur in properly tailored waveguides when a suitable orientation of the crystal optical axis, relative to the waveguide axis, is taken. The dependence of the allowed orientations on various waveguide parameters has been analyzed. Noticeable fast variations, with potential interest for switching applications, have been found.
\end{abstract}

$\mathrm{W}$ AVES guided by thin birefringent films have been extensively studied since the advent of integrated optics, due to the fact that the involved technology usually employs uniaxial dielectric materials (e.g., $\mathrm{LiNbO}_{3}$ and $\mathrm{LiTaO}_{3}$ ). Dispersion properties of waveguide modes, including leaky guided waves, have been extensively analyzed, thus now they are well established (for a review, see [1], [2], and references therein). Our goal in this letter is to point out that waveguides made on low-loss birefringent materials allow an additional type of guided waves, which has not been noticed early. For illustrative purposes, we pay attention here to a thin film of thickness $D$ of an isotropic material with refractive index $n_{f}$, surrounded by an isotropic material with refractive index $n_{c}$ and a positive birefringent crystal, whose ordinary and extraordinary refractive indices are $n_{\mathrm{os}}$ and $n_{\text {es }}$. The optical axis of the crystal is assumed to lie on the guide plane, forming an angle $\theta$ with the waveguide axis, which corresponds to off-axis propagation in an $X$ - or $Y$-cut sample.

Integrated optical technology typically employs materials for which $n_{\mathrm{es}}>n_{\mathrm{os}}>n_{\mathrm{c}}$ (e.g., $\mathrm{LiTaO}_{3}$-based waveguides with a $\mathrm{SiO}_{2}$ or glass buffer layer). Nevertheless, our aim here is to point out that when the involved materials verify $n_{\mathrm{es}}>n_{c}>n_{\mathrm{os}}$, the resulting structures allow existence of guided waves for waveguide parameters below usual cutoff. Above cutoff these waves become the well-known hybrid guided modes, and for $D / \lambda \rightarrow 0$, with $\lambda$ being the wavelength of the used radiation, they become the surface waves guided by the interface between the

Manuscript received August 28, 1992; revised October 13, 1992. This work was supported by the Comisión Interministerial de Ciencia Technología of the Spanish Government under Grant TIC92-0094-C0202.

L. Torner and J. P. Torres are with the Department of Signal Theory and Communications, Polytechnic University of Catalonia, Barcelona, Spain.

D. Mihalache is with the Department of Theoretical Physics, Institute of Atomic Physics, Bucharest, Romania.

IEEE Log Number 9206443 dielectric materials forming the substrate and the cover, which have been pointed out by Dyakonov [3]. In our calculations we take the parameters of a Corning 7059 glass film ( $n \simeq 1.57)$, deposited over a crystal quartz substrate $\left(n_{o} \simeq 1.544, n_{e} \simeq 1.553\right.$ ), with both a Corning 0041 glass $(n \simeq 1.545)$ and a low-alkali, lime-alumina borosilicate $(n \simeq 1.548)$ forming the cover, operating at $\lambda=589.3$ $\mathrm{nm}$.

With the exception of on-axis propagation configurations, waves guided by such an structure are of the hybrid type, with the six field components. Assuming timeharmonic dependence, $\vec{E}(\vec{r})=\vec{E}(z) \exp [j(\beta x-\omega t)]$, the fields in the substrate region are a superposition of ordinary and extraordinary waves, hence

$$
\begin{aligned}
\vec{E}_{s}(z)=\vec{E}_{\mathrm{os}} \exp \left[k_{0} \gamma_{\mathrm{os}}(D-z)\right] & \\
& +\vec{E}_{\mathrm{es}} \exp \left[k_{0} \gamma_{\mathrm{es}}(D-z)\right] .
\end{aligned}
$$

The transversal propagation constants of these evansecent fields are

$$
\begin{gathered}
\gamma_{o s}=\left(N^{2}-n_{o s}^{2}\right)^{1 / 2} \\
\gamma_{e s}=\frac{n_{e s}}{n_{e s}(\theta)}\left(N^{2}-n_{e s}^{2}(\theta)\right)^{1 / 2}
\end{gathered}
$$

with $N \equiv \beta / k_{0}$ being the effective index and $n_{\mathrm{es}}(\theta)=$ $n_{\mathrm{os}} n_{\mathrm{es}} / \sqrt{n_{\mathrm{os}}^{2} \sin ^{2} \theta+n_{\mathrm{es}}^{2} \cos ^{2} \theta}$. In the isotropic cover these solutions degenerate and one has $\vec{E}_{c}(z)=$ $\vec{E}_{c} \exp \left[k_{0} \gamma_{c} z\right]$, with

$$
\gamma_{c}=\left(N^{2}-n_{c}^{2}\right)^{1 / 2} .
$$

The eigenvalue equation for guided solutions is obtained by jointing these evanescent fields with the known field solution in the film region. It can be formally written as

$$
F(N ; \theta, \nu)=0
$$

where $\nu$ stands for all the involved waveguide parameters. The explicit expression of $F(N)$ is rather cumbersome and can be found in [4]. According to (2)-(4), stationary guided modes are obtained as roots of (5) verifying $N>$ $n_{c}, N>n_{\text {os }}$ and $N>n_{\mathrm{es}}(\theta)$. When $n_{\mathrm{es}}>n_{\mathrm{os}}>n_{c}$, exis- 
tence of guided modes is governed by the ordinary cutoff and the extraordinary cutoff, both to the substrate. In the first case, $N=n_{\mathrm{os}}$ and the ordinary wave forming the guided mode is radiated to the substrate, whereas in the second case, $N=n_{\mathrm{es}}(\theta)$ and the radiated wave has an extraordinary nature. At $\theta=0^{\circ}$, both conditions coincide and yield the cutoff point of the TE, TM-polarized guided modes. For $\theta \neq 0^{\circ}$, one has $n_{\text {es }}(\theta)>n_{\text {os }}$, therefore guided propagation requires $N>n_{\mathrm{es}}(\theta)$. Usual waveguide modes fulfill this requirement, but, as it is well-known, there is a cutoff thickness (in units of $\lambda$ ) for the guided waves to be allowed. Let us turn to the case $n_{\mathrm{es}}>n_{c}>n_{\mathrm{os}}$. Now, guided solutions occur above both the extraordinary-cutoff to the substrate and the usual cutoff to the $\operatorname{cover}\left(N=n_{c}\right)$, thus guided propagation requires $N>n_{\mathrm{es}}(\theta)$ and $N>n_{c}$. The point is that such kind of roots of (5) exist for any value of the ratio $D / \lambda$, provided that a suitable orientation of the crystal optical axis is taken. As it was noted by Dyakonov [3], this is true even for $D / \lambda=0$, then the waves being guided by the cover substrate dielectric interface. We will focus now on the existence conditions for guided waves below usual cutoff.

The allowed regions in the $\nu-\theta$ plane for stationary guided propagation to occur are obtained by drawing on it the critical optical axis orientations which yield the cutoff loci. These loci come from the conditions

$$
F\left(N=n_{c} ; \theta=\theta_{c 1}, \nu\right)=0
$$

and

$$
F\left(N=n_{e s}(\theta) ; \theta=\theta_{c 2}, \nu\right)=0 .
$$

In Figs. 1 and 2 we have plotted the allowed regions as a function of the ratio $D / \lambda$, for the two above-mentioned cover materials. At $D / \lambda=0$, the allowed angular interval is nearly centered at $\theta_{0}$, which is the orientation verifying $n_{\text {es }}\left(\theta_{0}\right)=n_{c}$. This interval is extremely narrow, $\Delta \theta=\theta_{c 2}$ $-\theta_{c 1} \simeq 2^{\circ} \times 10^{-3}$, although it encreases fast with $D / \lambda$. As $D / \lambda$ grows, $\theta_{c 1} \rightarrow 0^{\circ}$ and $\theta_{c 2} \rightarrow 90^{\circ}$. The limiting values are reached at the cutoff point of the $\mathrm{TE}_{0}$ wave taking place at these symmetric orientations. At $\theta=0^{\circ}$, the $\mathrm{TE}_{0}$ mode behaves as a purely ordinary wave, therefore

$$
\left.\frac{D}{\lambda}\right|_{\text {cutoff }}=\frac{1}{2 \pi \sqrt{n_{f}^{2}-n_{c}^{2}}} \tan ^{-1} \sqrt{\frac{n_{c}^{2}-n_{\mathrm{os}}^{2}}{n_{f}^{2}-n_{c}^{2}}} .
$$

Conversely, at $\theta=90^{\circ}$, the $\mathrm{TE}_{0}$ mode behaves as a purely extraordinary wave, hence

$$
\left.\frac{D}{\lambda}\right|_{\text {cutoff }}=\frac{1}{2 \pi \sqrt{n_{f}^{2}-n_{\mathrm{es}}^{2}}} \tan ^{-1} \sqrt{\frac{n_{\mathrm{es}}^{2}-n_{c}^{2}}{n_{f}^{2}-n_{\mathrm{es}}^{2}}} .
$$

Above these cutoff values, usual hybrid waveguide modes take place. Finally, the allowed region in the $n_{c}-\theta$ plane for stationary guided propagation are shown in Figs. 3 and 4 , for film thicknesses $D / \lambda=0.2$ and $D / \lambda=0.3$, respectively. The plots show noticeable fast variations of $\theta_{c 1}$ and $\theta_{c 2}$ as a function of $n_{c}$.

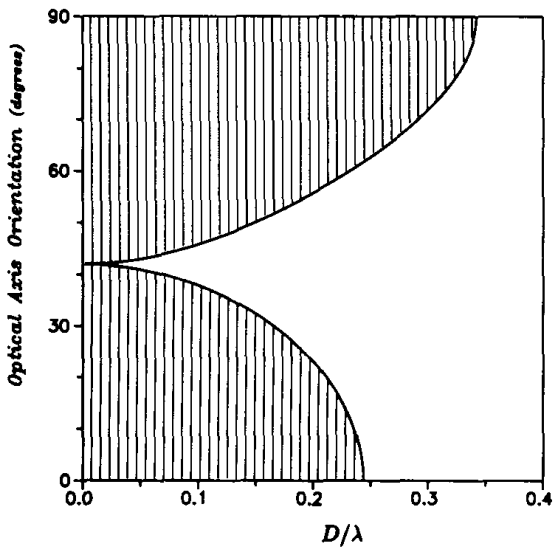

Fig. 1. Allowed optical axis orientations for stationary guided propagation to occur as a function of the ratio $D / \lambda$. Dashed regions: forbidden values. Here $n_{c}=1.548$.

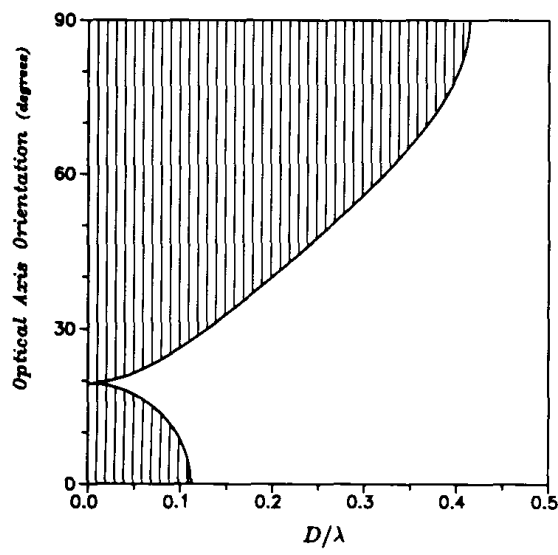

Fig. 2. Same as in Fig. 1, but for $n_{c}=1.545$.

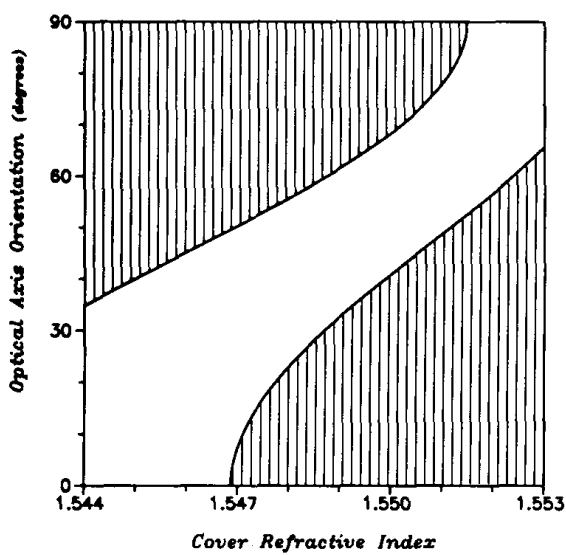

Fig. 3. Allowed optical axis orientations as a function of the cover refractive index. Here $D / \lambda=0.2$. 


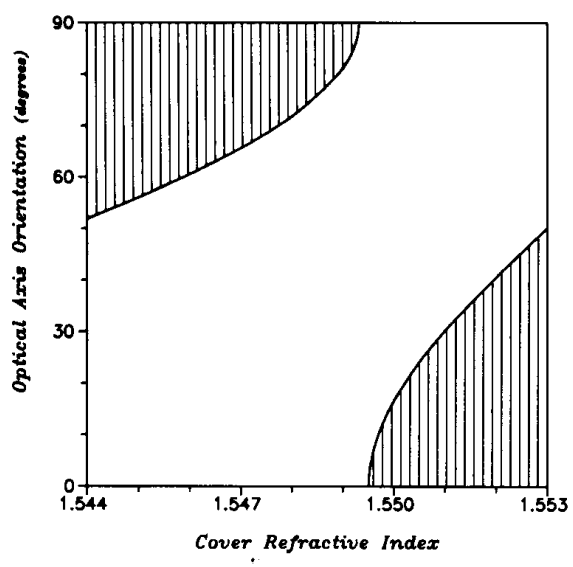

Fig. 4. Same as in Fig. 3, but for $D / \lambda=0.3$.

To sum up, the main conclusion of Figs. 1-4 is that existence conditions of the reported guided waves strongly depend on the waveguide parameters. This behavior sug- gests that the related guided-to-radiated mode transition can be useful for switching applications requiring strong sensitive dependence on the refractive index values. Thermooptically induced variations, by pulse heating, and intensity-dependent refractive-index changes in properly tailored waveguides made on optically nonlinear materials are possibilities to be investigated [5], [6].

\section{REFERENCES}

[1] A. Knoesen, T. K. Gaylord, and M. G. Moharam, "Hybrid guided modes in uniaxial dielectric planar waveguides," J. Lightwave Technol., vol. LT-6, pp. 1083-1104, 1988.

[2] T. K. Gaylord and A. Knoesen, "Passive integrated optical anisotropy-based devices," J. Mod. Opt., vol. 35, pp. 925-946, 1988.

[3] M. I. D'yakonov "New type of electromagnetic wave propagating at an interface," Sov. Phys. JETP, vol. 67, pp. 714-716, 1988.

[4] L. Torner, F. Canal, and J. Hernandez-Marco, "Leaky modes in multilayer uniaxial optical waveguides," Appl. Opt., vol. 29, pp. 2805-2814, 1990.

[5] I. C. Khoo, P. Zhou, R. R. Michael, R. G. Lindquist, and R. Mansfield, "Optical switching by a dielectric-cladded nematic film," IEEE J. Quantum Electron., vol. QE-25, pp. 1755-1759, 1989.

[6] J. Paškauskas and D. Čiplys, "Optical waveguiding in thermooptically perturbed surface layers," Opt. Commun., vol. 91, pp. 46-50, 1992.

\title{
Voltage-Controlled $N \times N$ Coupled Waveguide Switch and Power Splitter
}

\author{
S. Ruschin and D. Meshulach
}

\begin{abstract}
It is shown that it is possible to accomplish nearcomplete power transfer functions in $N$ coupled waveguide arrays by controlling a set of $N$ voltages applied to the waveguides. Switching of the power from an excited center waveguide into any output channel in a five waveguide array, and splitting of power into any output combination of channels is demonstrated. In all cases, the crosstalk is better than $-16 \mathrm{~dB}$, and the power splitting efficiency ratio differs from 1 on an amount of not more than $15 \%$. The length of the device is arbitrarily set. The sensitivity of the splitting functions to small random changes of the applied voltages is investigated.
\end{abstract}

\section{INTRODUCTION}

$\mathrm{O}$ PTICAL switching devices have long been of interest in the field of optoelectronics [1]. Two-arm direc-

Manuscript received August 11, 1992; revised November 9, 1992. S. Ruschin is with the Faculty of Engineering, Department of Electrical Engineering, Physical Electronics, Tel-Aviv 69978, Israel.

D. Meshulach is with the School of Physics and Astronomy, Tel-Aviv University, Tel-Aviv 69978, Israel.

IEEE Log Number 9206987. tional couplers have become important in integrated optics, serving as routing elements, and as sub-elements in more complicated devices. Three-arm directional couplers were also analyzed, and shown to be useful in power transfer between the outermost guides, and in sampling and filtering [2], [3]. Multiple coupled waveguide systems were considered later on, and found capable of performing a variety of operating functions. Particular devices of interest are $N \times N$ optical switches, and high-power semiconductor laser arrays [4], [5].

For $N \times N$ coupled waveguide switches, it was shown, that for a system of synchronous waveguides, symmetric coupling coefficients can be chosen so that power can be completely transferred form one outermost waveguide to the other outermost waveguide. It was also demonstrated that in systems with an odd number of waveguides, excitation of the center waveguide can be fully transferred to the outer waveguides and vice versa [4], [6], [7]. In order to accomplish complete power transfer from any waveguide at the input to any other at the output, it was realized by 